\title{
Pre-treatment of surface waters for ceramic microfiltration
}

D. Metcalfe ${ }^{1}$, P. Jarvis ${ }^{2}$, C. Rockey ${ }^{1}$, S. Judd ${ }^{2,3^{*}}$

${ }^{1}$ South West Water, Exeter, UK. ${ }^{2}$ Cranfield University, Cranfield, Beds, UK. ${ }^{3}$ Qatar University, Doha, Qatar. *Corresponding author, s.j.judd@ cranfield.ac.uk.

\author{
Abbreviations: \\ BBs - Building blocks \\ BPs - Biopolymers \\ BW - Backwash \\ CEB - Chemically enhanced backwash \\ CIP - Cleaning in place \\ $\mathrm{CMF}$ - Ceramic microfiltration \\ DOC - Dissolved organic carbon \\ HMW - High molecular weight \\ HSs - Humic substances \\ IEX - Ion exchange \\ ILCA - Inline coagulation \\ IR - Irreversible \\ LC-OCD - Liquid chromatography-organic carbon detection \\ LORIV - Lowland river (River Tamar) \\ LMW - Low molecular weight \\ LMW-N - Low molecular weight-neutrals \\ NOM - Natural organic matter \\ NTU - Nephelometric turbidity unit \\ $\mathrm{PACl}$ - poly-aluminium chloride \\ SIX - Suspended Ion exchange \\ TMP - Trans-membrane pressure \\ UPRES - Upland reservoir (Burrator Reservoir) \\ UPRIV - Upland river (River Tavy) \\ UVT - Filtered UV transmittance at $254 \mathrm{~nm}$ \\ WTWs - Water treatment works
}

\section{Abstract}

The influence of pre-treatment on the suppression of irreversible (IR) fouling of ceramic membranes challenged with three UK surface waters has been studied at pilot scale. An initial scoping study compared the efficacy of suspended ion exchange (SIX) and clarification (coagulation followed by sludge blanket clarification) individually and in combination. Direct membrane filtration following in-line coagulation (ILCA) was also investigated with and without SIX. The impact on the various organic fractions, specifically high molecular weight (HMW) biopolymers (BPs) and humic substances (HSs), and lower molecular weight (LMW) building blocks (BBs) and neutrals, was studied using liquid chromatography - organic carbon detection (LC-OCD).

Results revealed SIX and coagulation to preferentially remove the LMW and HMW organic fractions respectively. Residual HMW organic matter (primarily BPs) following SIX pretreatment were retained by the membrane which led to rapid irreversible fouling. Coagulation pre-treatment provided stable membrane operation and the residual LMW organics were not significantly retained by the membrane. Combining clarification and SIX resulted in 
significantly increased removal of organics and lower membrane fouling rates. Tests performed using SIX and ILCA revealed comparably high dissolved organic carbon (DOC) removal to SIX with clarification. However, unlike the case for clarification with SIX, the addition of SIX to optimised ILCA dosing offered no additional suppression of membrane fouling compared to ILCA alone. Optimised ILCA pretreatment led to very low IR fouling rates of $<0.3 \mathrm{kPa} / \mathrm{day}$ trans-membrane pressure, despite highly challenging operating conditions of elevated fluxes $\left(185 \mathrm{~L} \mathrm{~m}^{-2} \mathrm{~h}^{-1}\right)$ and highly variable feedwater dissolved organic carbon concentrations.

Keywords: Ceramic membrane; pretreatment; ion exchange; coagulation; organic fouling

\section{Introduction}

Historically, the conventional processes of coagulation, clarification and granular media filtration have been used for removing suspended material and natural organic matter (NOM) from surface water prior to disinfection (Bond et al, 2011). Membrane filtration offers some key advantages over such processes, including higher removal efficiency, compactness, robustness against fluctuating feed water quality, and the provision of an absolute barrier against suspended particles and pathogens such as Cryptosporidium (Huang et al. 2009; Vreeburg et al. 2008). Membranes have thus been increasingly applied for water treatment (Huang et al, 2012), with polymeric materials being most commonly used.

Recently there has been increased interest in ceramic membranes for potable and industrial water treatment applications due to their greater operational lifetime, solids loading capacity, sustainable flux rates (from reduced organic fouling), mechanical robustness and resistance to aggressive cleaning protocols (Hofs et al., 2011; Lee \& Kim, 2014). These and other technological benefits have meant that the higher capital costs associated with ceramic membranes can be significantly offset by lower operating costs over the life of the installation, making ceramic membranes potentially economically competitive on a whole life cost basis (Freeman and Shorney-Darby, 2011; Meyn et al. 2012; Shang et al. 2015).

Membrane fouling nonetheless remains a major obstacle to the application of membranes per se. Much research has been focussed on understanding fouling mechanisms and identifying pretreatment capable of removing highly-fouling compounds (Huang et al. 2009). Such research has generally revealed that the high molecular weight (HMW) biopolymer (BP) fraction of NOM is primarily responsible for irreversible (IR) membrane fouling, i.e. demanding intensive chemical cleaning in place (Fan et al. 2008; Tian et al. 2013; Kimura et al. 2014). Pretreatment methods which substantially remove the BP fraction have been shown to provide stable membrane operation, with coagulation being the most consistently successful method (Fabris et al. 2007; Huang et al. 2012; Humbert et al. 2007). Coagulation preferentially flocculates the HMW organics, including BPs. The resulting solids are then either removed by clarification (e.g. sludge blanket clarification or dissolved air flotation) or, where "in-line" coagulation with direct filtration is used, by the backwash cycle of the membrane process.

Adsorption processes (anion exchange and activated carbon) have been shown to improve treated water quality through the removal of low molecular weight (LMW) organics but, since they remove only small amounts of the HMW fraction, in most cases fouling reduction has been shown to be minimal (Humbert et al. 2007; Fabris et al. 2007; Fan et al. 2008; Huang et al. 2012). Against this, some studies have revealed LMW organics to cause or contribute to fouling through synergistic action with the higher MW fraction (Gray et al. 2011; Subhi et al. 
2012; Lai et al. 2015). Such differences in findings highlight the complexity of interactions between the organic constituents and the membrane material and fouling layer.

Since coagulation and adsorption, and specifically ion exchange processes (IEX), have been shown to preferentially remove the high and low MW fractions of the NOM respectively, it may be surmised that their use in combination may both improve permeate water quality and suppress membrane fouling. However, previous studies of the use of combined IEX and coagulation upstream of membrane filtration have not unequivocally demonstrated membrane fouling benefits: reported fouling rates have been similar to those possible with coagulation alone (Humbert et al. 2007; Fan et al. 2008).

The current study aimed to evaluate suppression of irreversible fouling of ceramic membranes associated with pretreatment by suspended ion exchange (SIX), coagulation or a combination of both. The analysis proceeded through quantification of the organic fractions removed by both pretreatment and the membrane itself, and examined the resulting impact on membrane fouling. Experiments were conducted for three UK surface waters at large pilot scale over a 26 month period under conditions appropriate for potable water production. The irreversible fouling rate was assessed from trans-membrane pressure (TMP) transients generated under operating conditions (including physical and chemically enhanced backwashing) pertaining to those applied at full-scale.

\section{Methods and materials}

\subsection{Raw waters}

Three raw waters were tested, either individually or as a blend:

- An upland reservoir (UPRES) of low turbidity and low-to-moderate dissolved organic carbon (DOC) (Burrator Reservoir).

- A soft, upland river (UPRIV), prone to rapid changes in quality following rain, of low-tohigh DOC and low-to-moderate turbidity (River Tavy).

- A lowland river (LORIV) prone to rapid changes in quality following rain of low-to-high DOC and turbidity (River Tamar).

\subsection{Pilot plant}

The $150 \mathrm{~m}^{3} /$ day pilot plant (Fig. 1) comprised the SIX ${ }^{\circledR}$ and ceramic membrane filtration $(\mathrm{CMF})(\mathrm{CeraMac} \AA)$ processes and subsequently the SIX®, in-line coagulation (ILCA®) and CMF (PWN Technologies, Netherlands). These processes have been described elsewhere (Galjaard et al. 2011; Metcalfe et al. 2015) and feature:

- SIX®: an acrylic quaternary amine, gel-type strongly basic anion exchange resin in the chloride form was used throughout the trial (Lewatit S5128, Lanxess, Germany). The resin was generally dosed at $18 \mathrm{~mL} / \mathrm{L}$ with a contact time of $30 \mathrm{~min}$, with dosing conditions informed by preliminary bench-scale tests. Tests were also performed with lower or zero resin doses (i.e. with ILCA $®$ only) as appropriate. The resin was in continuous use over the 2 years of the trial.

- A Lamella separator was used for separating the resin from the treated flow and the resin regenerated with $30 \mathrm{~g} / \mathrm{L} \mathrm{NaCl}$. SIX-treated water samples were collected directly after resin separation.

- ILCA® using polyaluminium chloride (WAC®, Water Treatment Solutions, UK, 0.53$4.23 \mathrm{mg} / \mathrm{L}$ as $\mathrm{Al}$ ) was used alone or following SIX pretreatment. Water was $\mathrm{pH}$-corrected with $\mathrm{NaOH}$ or $\mathrm{HCl}$ (Brenntag, U.K), injected with coagulant, and mixed by a static mixer 
and flocculated for 2.4-3.9 minutes prior to CMF (direct filtration). The coagulation $\mathrm{pH}$ was circa 6.4 for all tests.

- CMF was carried out using a vertically mounted $25 \mathrm{~m}^{2}$ ceramic membrane element (Metawater, Japan, nominal pore size $0.1 \mathrm{~mm}$ ) operating by dead end filtration.

\subsection{Full scale WTW process}

The water treatment works ((WTWs) Crownhill WTWs, South West Water (SWW), Plymouth, U.K) treated water using optimised coagulation with aluminium sulphate dosed at 3.39$6.36 \mathrm{mg} / \mathrm{L}$ as $\mathrm{Al}$ (Kemira, U.K) and Magnafloc LT25 (BASF, U.K) anionic polyelectrolyte at 0.1-0.2 mg/L. Powdered activated carbon (PAC, Aquasorb BP2, Jacobi, U.K) was dosed at 2$3 \mathrm{mg} / \mathrm{L}$ prior to coagulation. The WTWs coagulant dose was optimised through jar testing and works operation. Clarified water following flash mixing, flocculation and solids-liquid separation (by sludge blanket clarification) was supplied to the pilot plant for some of the tests.

\section{$2.4 \quad$ Pretreatment}

The pilot plant was fed with either raw or, during the clarification or clarification + SIX campaigns, clarified water (Fig. 1).

Tested pretreatment options for CMF comprised:

1) Clarification only, or clarification followed by SIX: Raw water was treated by the full scale clarification process and the water piped from the clarifier outlets to the pilot plant feed tank. During clarification-only tests the SIX process was taken off-line and all resin removed. Additional tests were performed where the clarified water was further treated by SIX within the pilot plant, prior to CMF.

2) SIX followed by ILCA, or ILCA alone: ILCA was employed following the SIX process, or when ILCA was used alone the SIX process was taken off line and the resin removed. For most tests the coagulant dose and $\mathrm{pH}$ correction was manually controlled, which led to periods of sub-optimal operation due to the rapidly changing raw water sources. The $\mathrm{pH}$ and coagulant dosing systems were automated in March 2015.

\subsection{Ceramic microfiltration (CMF)}

The CMF membrane flux was varied between 109 and $250 \mathrm{LMH}\left(\mathrm{L} /\left(\mathrm{m}^{2} \mathrm{~h}\right)\right)$. A new membrane was installed at the start of the trials in March 2013, replaced by a new element after 2 years in operation (March 2015) prior to extended tests with automated coagulant and $\mathrm{pH}$ control.

Backwashing and chemically enhanced backwashing (BW and CEB) were performed following a given filtration load, measured in litres of feed solution filtered per $\mathrm{m}^{2}$ membrane surface $\left(\mathrm{L} / \mathrm{m}^{2}\right)$. BW used $75 \mathrm{~L}$ permeate pressurised to 5 bar and passed through the membrane in a reverse flow direction. CEBs with $\mathrm{NaOCl}(100 \mathrm{mg} / \mathrm{L}), \mathrm{NaOH}(\mathrm{pH} 12)$ or acid $+\mathrm{H}_{2} \mathrm{O}_{2}(\mathrm{pH}$ $2.5,100 \mathrm{mg} / \mathrm{L} \mathrm{H}_{2} \mathrm{O}_{2}$ ) were performed at 2 bar pressure. The membrane was soaked in the reagent for 10 minutes prior to a standard BW. Process conditions for the tests are reported in Table 1. 


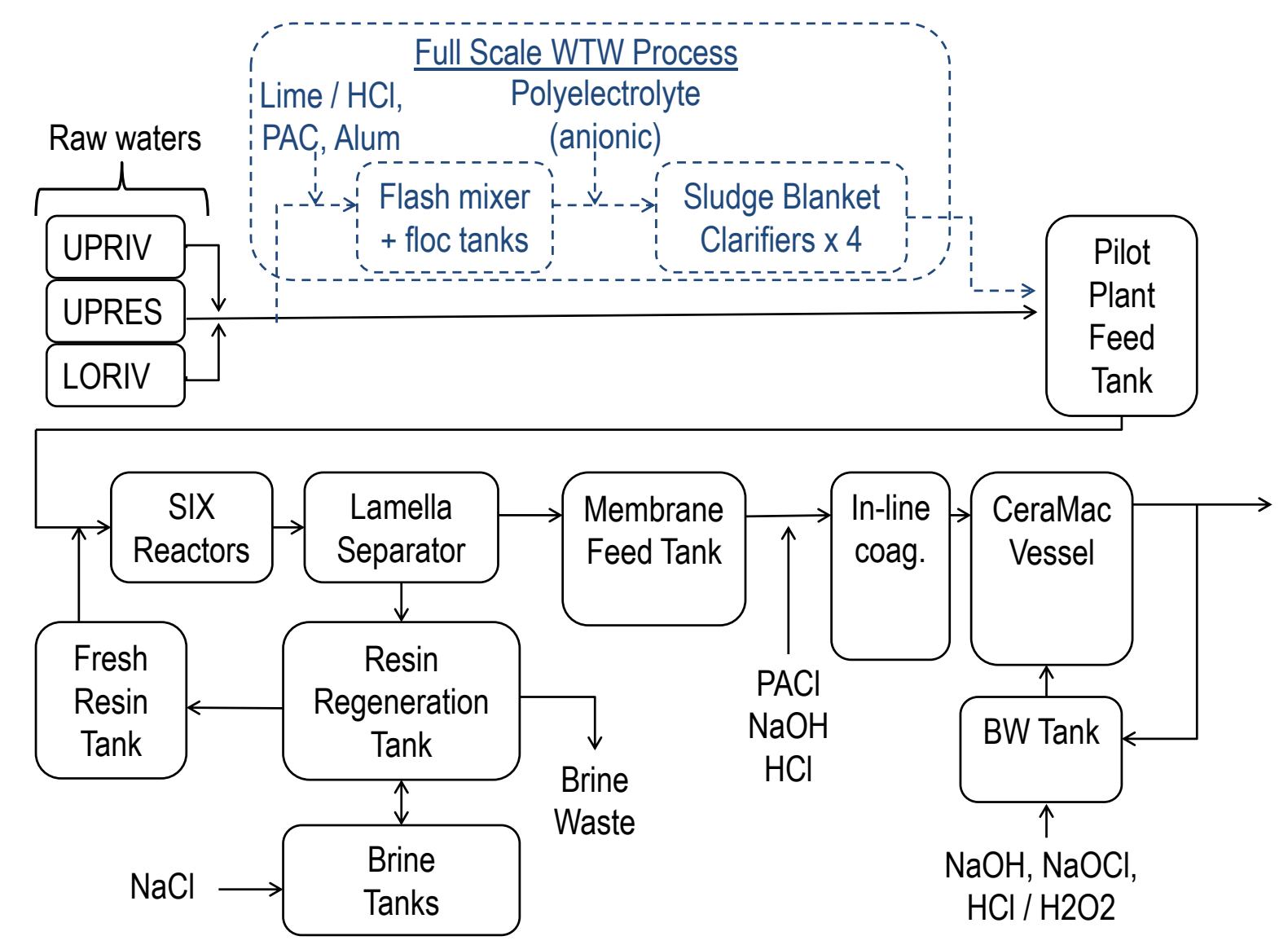

Figure 1: Process flow diagram

After each discrete test cleaning in place (CIP) was performed by circulating chemical solution through the membrane for an extended period of time ( $3-24$ hours). Generally an overnight CIP using $\mathrm{NaOH}$ ( $\mathrm{pH} 12-12.5$ ) was performed, followed by a CIP with either $\mathrm{NaOCl}$ (100-500 $\mathrm{mg} / \mathrm{L})$ or $\mathrm{HCl} / \mathrm{H}_{2} \mathrm{O}_{2}\left(\mathrm{pH} 2.7,100 \mathrm{mg} / \mathrm{L} \mathrm{H}_{2} \mathrm{O}_{2}\right)$ if required to increase the specific flux to $>300$ $\mathrm{LMH} /$ bar at $10^{\circ} \mathrm{C}$ prior to starting a new test. This low specific flux target (300 LMH/bar) did not constitute an optimised recovery of permeability but provided an attainable standard starting permeability for each test, despite the significant fouling which occurred in some of the scoping trials. Following the final extended tests, based on a virgin membrane with automated coagulant and $\mathrm{pH}$ control, an intensive CIP was performed which fully recovered membrane permeability (to $\sim 1250 \mathrm{LMH} / \mathrm{bar}$ at $10^{\circ} \mathrm{C}$ ). The conditions for the intensive CIP were 30 minutes at $\mathrm{pH} 2.7$ with $100 \mathrm{mg} / \mathrm{l} \mathrm{H} 2 \mathrm{O} 2$, followed by 1 hour at $\mathrm{pH} 12(\mathrm{NaOH})$, followed by $2 \% \mathrm{NaOCl}$ for 3 days. 
Table 1: Test process conditions. * Backwash (BW) and chemically enhanced BW (CEB) sequences have been described as, for example, (5 BW, NaOCl CEB) x 4, $5 \mathrm{BW}, \mathrm{HCl} / \mathrm{H}_{2} \mathrm{O}_{2} \mathrm{CEB}$ - this would denote $5 \mathrm{BW}$ followed by a $\mathrm{NaOCl} \mathrm{CEB}$ with this sequence being repeated 4 times. Following this $5 \mathrm{BW}$ and then an $\mathrm{HCl} / \mathrm{H}_{2} \mathrm{O}_{2} \mathrm{CEB}$ would occur and then the sequence would be repeated.

\begin{tabular}{|c|c|c|c|c|c|c|}
\hline $\begin{array}{l}\text { Test } \\
\text { No. }\end{array}$ & $\begin{array}{l}\text { Raw water } \\
\text { source }\end{array}$ & Pretreatment & $\begin{array}{l}\text { Flux } \\
(\text { LMH })\end{array}$ & $\begin{array}{c}\text { Filtration load } \\
\text { prior to } B W / C E B \\
\left(L / m^{2}\right)\end{array}$ & $\begin{array}{c}B W \text { number prior to } C E B / \\
C E B \text { sequence and chemicals } \\
*\end{array}$ & $\begin{array}{c}\text { Water } \\
\text { recovery } \\
(\%)\end{array}$ \\
\hline 1 & UPRES & SIX & 150 & $75 / 450$ & $\begin{array}{c}(5 \mathrm{BW}, \mathrm{NaOCl} \mathrm{CEB}) \times 4,5 \\
\mathrm{BW}, \mathrm{HCl} / \mathrm{H}_{2} \mathrm{O}_{2} \mathrm{CEB}\end{array}$ & 95.3 \\
\hline 2 & UPRES & SIX & 150 & $75 / 450$ & $\begin{array}{c}(5 \mathrm{BW}, \mathrm{NaOCl} \mathrm{CEB}) \times 4,5 \\
\mathrm{BW}, \mathrm{HCl} / \mathrm{H}_{2} \mathrm{O}_{2} \mathrm{CEB}\end{array}$ & 95.3 \\
\hline 3 & $\begin{array}{l}\text { UPRIV / } \\
\text { UPRES }\end{array}$ & $\begin{array}{l}\text { Clarification } \\
\text { only }\end{array}$ & 150 & $75 / 450$ & $\begin{array}{c}(5 \mathrm{BW}, \mathrm{NaOCl} \mathrm{CEB}) \times 4,5 \\
\mathrm{BW}, \mathrm{HCl} / \mathrm{H}_{2} \mathrm{O}_{2} \mathrm{CEB}\end{array}$ & 95.3 \\
\hline 4 & $\begin{array}{l}\text { LORIV/UPRIV/ } \\
\text { UPRES }\end{array}$ & $\begin{array}{l}\text { Clarification / } \\
\text { SIX }\end{array}$ & 150 & $75 / 450$ & $\begin{array}{c}(5 \mathrm{BW}, \mathrm{NaOCl} \mathrm{CEB}) \times 4,5 \\
\mathrm{BW}, \mathrm{HCl} / \mathrm{H}_{2} \mathrm{O}_{2} \mathrm{CEB}\end{array}$ & 95.3 \\
\hline 5 & UPRES & SIX + ILCA & 185 & $100 / 1800$ & $\begin{array}{c}\text { 17BW, } \mathrm{NaOH} \text { CEB, } \\
\mathrm{HCl} / \mathrm{H}_{2} \mathrm{O}_{2} \mathrm{CEB}\end{array}$ & 96.8 \\
\hline 6 & UPRES & SIX + ILCA & 185 & $100 / 1800$ & $\begin{array}{c}17 \mathrm{BW}, \mathrm{NaOH} \mathrm{CEB}, \\
\mathrm{HCl} / \mathrm{H}_{2} \mathrm{O}_{2} \mathrm{CEB}\end{array}$ & 96.8 \\
\hline $6 a$ & UPRES & SIX + ILCA & 185 & $100 / 1800$ & $\begin{array}{c}17 \mathrm{BW}, \mathrm{NaOH} \mathrm{CEB}, \\
\mathrm{HCl} / \mathrm{H}_{2} \mathrm{O}_{2} \mathrm{CEB}\end{array}$ & 96.8 \\
\hline 7 & UPRIV & SIX + ILCA & 185 & $100 / 1800$ & $\begin{array}{c}17 \mathrm{BW}, \mathrm{NaOH} \mathrm{CEB}, \\
\mathrm{HCl} / \mathrm{H}_{2} \mathrm{O}_{2} \mathrm{CEB}\end{array}$ & 96.8 \\
\hline 8 & LORIV & SIX + ILCA & 185 & $100 / 1800$ & $\begin{array}{c}17 \mathrm{BW}, \mathrm{NaOH} \mathrm{CEB} \\
\mathrm{HCl} / \mathrm{H}_{2} \mathrm{O}_{2} \mathrm{CEB}\end{array}$ & 96.8 \\
\hline 9 & UPRES & ILCA Only & 109 & $150 / 1800$ & $\begin{array}{l}(17 \mathrm{BW}, \mathrm{NaOH}) \times 3,17 \mathrm{BW} \\
\mathrm{NaOH} \mathrm{CEB}, \mathrm{HCl} / \mathrm{H}_{2} \mathrm{O}_{2} \mathrm{CEB}\end{array}$ & 97.8 \\
\hline 10 & UPRES & $\begin{array}{l}\text { 9ml/l SIX + } \\
\text { ILCA }\end{array}$ & 109 & $150 / 1800$ & $\begin{array}{l}(17 \mathrm{BW}, \mathrm{NaOH}) \times 3,17 \mathrm{BW} \\
\mathrm{NaOH} \mathrm{CEB}, \mathrm{HCl} / \mathrm{H}_{2} \mathrm{O}_{2} \mathrm{CEB}\end{array}$ & 97.8 \\
\hline 11 & UPRES & $\begin{array}{l}\text { 18ml/1 SIX + } \\
\text { ILCA }\end{array}$ & 109 & $150 / 1800$ & $\begin{array}{l}(17 \mathrm{BW}, \mathrm{NaOH}) \times 3,17 \mathrm{BW} \\
\mathrm{NaOH} \mathrm{CEB}, \mathrm{HCl} / \mathrm{H}_{2} \mathrm{O}_{2} \mathrm{CEB}\end{array}$ & 97.8 \\
\hline 12 & UPRES & ILCA Only & 109 & $150 / 1800$ & $\begin{array}{l}(17 \mathrm{BW}, \mathrm{NaOH}) \times 3,17 \mathrm{BW} \\
\mathrm{NaOH} \mathrm{CEB}, \mathrm{HCl} / \mathrm{H}_{2} \mathrm{O}_{2} \mathrm{CEB}\end{array}$ & 97.8 \\
\hline 13 & UPRES & $\begin{array}{l}18 \mathrm{ml} / \mathrm{l} \mathrm{SIX} \\
\text { Only }\end{array}$ & 109 & $150 / 1800$ & $\begin{array}{l}(17 \mathrm{BW}, \mathrm{NaOH}) \times 3,17 \mathrm{BW} \\
\mathrm{NaOH} \mathrm{CEB}, \mathrm{HCl} / \mathrm{H}_{2} \mathrm{O}_{2} \mathrm{CEB}\end{array}$ & 97.8 \\
\hline
\end{tabular}

\subsection{Coagulant dose optimisation}

20 jar tests were performed on a wide range of raw or SIX treated waters from each of the 3 sources to establish a means of optimising the coagulant dose for minimising membrane fouling. The data from these jar tests revealed an excellent correlation $\left(\mathrm{R}^{2}=0.96\right)$ between the optimum coagulant dose and feed water filtered UV transmittance (UVT), the optimum coagulant dose determined as being the minimum coagulant dose in $\mathrm{mg} / \mathrm{L}$ as $\mathrm{Al}$ providing maximum achievable UVT removal (Fig. 2):

$$
\mathrm{C}_{\text {opt }}=8.88-0.0911 * \mathrm{UVT}_{\text {feed }}
$$

Applications of this dose resulted in negligible membrane adsorption of organics according to liquid chromatography-organic carbon detection (LC-OCD) analysis and on-site membrane 
feed and permeate UVT measurements (Section 2.9). Most of the tests were conducted with daily manual adjustments to the coagulant dosing, based on the feed water UVT according to Equation 1, along with $\mathrm{pH}$ correction. This resulted in occasional sub-optimal coagulation conditions when rapid changes in raw water quality took place, subsequently leading to increased membrane fouling.

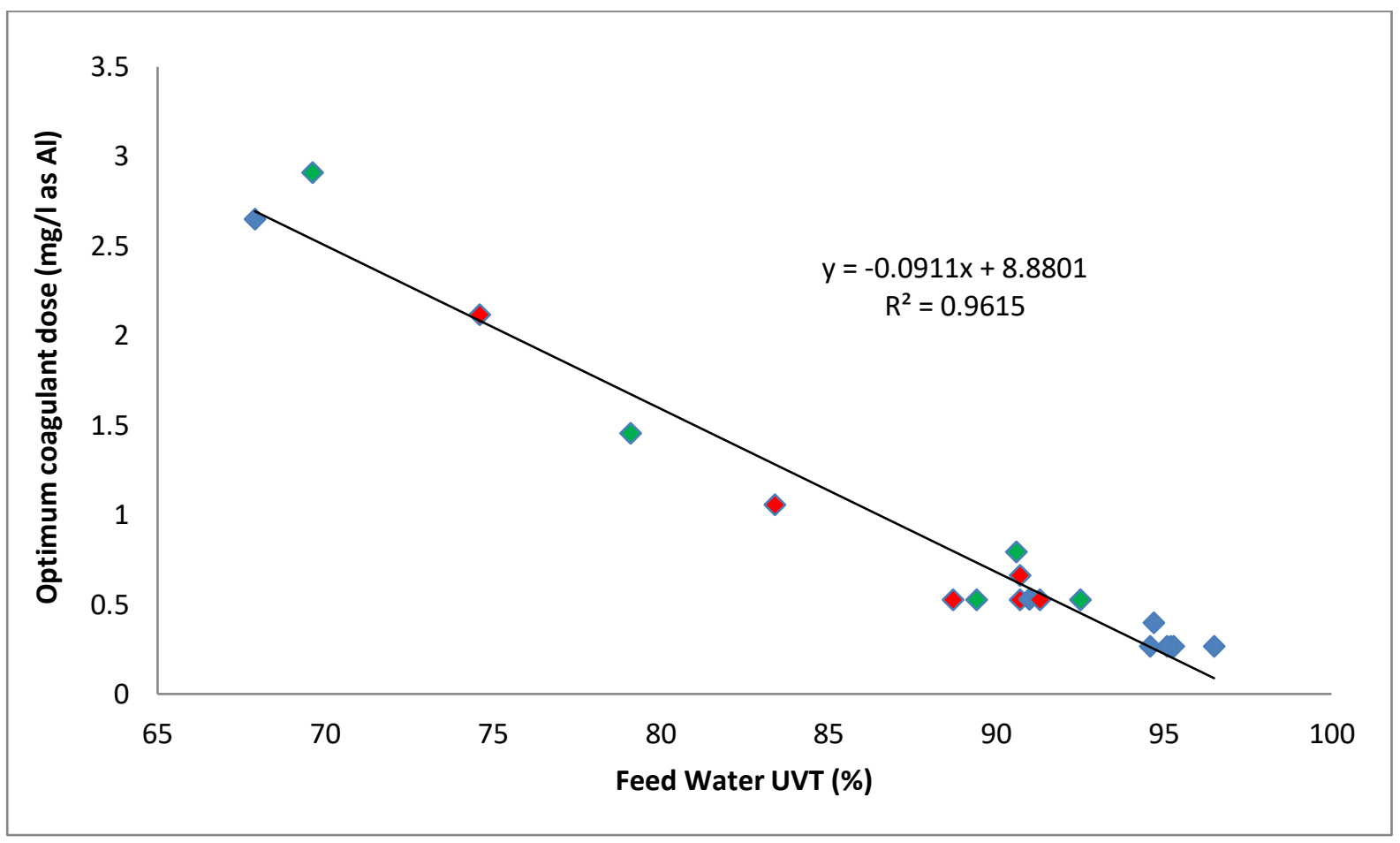

Figure 2: $\quad$ Feed water UVT vs. optimum coagulant dose jar tests results. Red $=$ UPRES, Blue $=$ UPRIV, Green $=$ LORIV

\subsection{Automated coagulant dosing and $\mathrm{pH}$ correction}

In March 2015 automated coagulant and pH control was installed. A Spectro::lyser (S::CAN, Austria) was used to measure the solids-compensated UVT of the water in the membrane feed tank, for which measured values were similar to manual filtered UVT samples. A controller used the on-line $\mathrm{UVT}_{\text {feed }}$ value with Equation 1 to provide the required coagulant dose. $\mathrm{pH}$ control was automated to control to a set-point of 6.4 via a negative feedback loop/controller.

\subsection{Membrane fouling rate}

Irreversible membrane fouling (i.e. not recovered by the routine BW and CEB) was measured so as to determine the required CIP frequency during full-scale operation. Individual filtration runs were generally based on a total filtration volume of $625 \mathrm{~m}^{3}$, the filtration run time then varying with flux. An extended run was performed with a virgin membrane, with automated coagulant and $\mathrm{pH}$ control. TMP and temperature data (logged at one minute intervals) from each test was analysed. TMP data following CEBs were selected and the values corrected to $10^{\circ} \mathrm{C}$. The fouling rate in $\mathrm{kPa} /$ day was determined by linear regression, with the corresponding $\mathrm{R}^{2}$ value (Table 2 ). 


\subsection{Sample analysis}

Turbidity was analysed using a Hach Lange 2100AN Turbidimeter (Hach Lange, Germany). $0.45 \mu \mathrm{m}$-filtered UVT was measured using a Hach DR6000 spectrophotometer (Hach Lange, Germany). DOC was characterised by LC-OCD analysis at Het Water Laboratorium (Netherlands), which quantified concentrations of total DOC and the discrete MW fractions of biopolymers (BP)s, humic substances (HSs), building blocks (BBs), LMW neutrals and LMW acids (Huber et al., 2011). LMW acids were not detected by LC-OCD analysis since organics within this band (50 minute elution time, equating to $<350$ Daltons) absorbed UV and were hence classified by the analysis/software as LMW-HS. Turbidity and LC-OCD results for each test are reported in Table 2.

Adsorption of DOC and the fractions thereof onto the membrane was assessed through concentration difference between the feed and permeate. Since UVT was found to correlate reasonably well $\left(\mathrm{R}^{2}=0.63-0.92\right)$ with DOC concentration, all source water organic fractions being strongly UV absorbing, UVT data were used to derive organic carbon values in those tests where no LC-OCD data was collected.

\section{Results}

\subsection{Scoping studies, SIX, clarification or clarification + SIX (Tests 1-4)}

A number of initial tests (Tests 1-4, Tables 1-2) were performed to establish the influence of pre-treatment on irreversible fouling. These tests compared SIX treatment alone, at pilot scale, with a full scale clarification process and a combined process where the clarified water was further treated by SIX, at $150 \mathrm{LMH}$. LC-OCD analysis illustrated clear differences in the organic fractions removed by SIX, clarification and a combined process of clarification followed by SIX (Fig. 3a-d). SIX pre-treatment preferentially removed LMW organics whilst the removal of HMW fractions by SIX was low, especially for the highest MW BP fraction (25-29\%). Conversely, coagulation pretreatment preferentially removed the highest MW fractions (particularly BP) whilst the LMW organics fractions were removed to a lesser extent than possible with SIX (especially lower MW HS and BB fractions) (Fig. 3c). These data are consistent with the findings of previous studies showing some LMW organics to be recalcitrant to coagulation and the removal of high MW BPs by IEX to be marginal (Fabris et al. 2007; Huber et al. 2011; Humbert et al. 2007; Mergen et al. 2009; Myat et al. 2012). Subsequently, clarification followed by SIX was found to yield very low residual organics concentrations (Fig. 3d), in keeping with previously reported findings (Fearing et al. 2004; Jarvis et al. 2008; Humbert et al. 2007; Fan et al. 2008; Singer and Bilyk, 2002).

In Tests 1 and 2, following SIX pre-treatment, the majority (58-80\%) of the residual HMW organics were retained by the membrane (Figs. 3a and b). At the lowest DOC levels (Table 2, Test 1 and Fig, 3a), a low-to-moderate fouling rate of $3.6 \mathrm{kPa} /$ day was obtained at $150 \mathrm{LMH}$. When DOC increased for the UPRES source and an organic compositional change occurred resulting in a near three-fold increase in the concentration of the HMW BP fraction, a very high membrane fouling rate (48 kPa/day) was recorded (Table 2, Test 2 and Fig. 3b). Further tests with SIX pre-treatment were performed on higher DOC reservoir and river water sources (UPRES, UPRIV and LORIV) at 100 and $150 \mathrm{LMH}$. Despite reductions in filtration volume prior to backwash and CEB, all of these tests yielded very high fouling rates (82-863 kPa/day).

Table 2: Test membrane irreversible fouling rates and water quality data. 


\begin{tabular}{|c|c|c|c|c|c|c|c|c|c|c|c|c|}
\hline \multirow[b]{2}{*}{$\begin{array}{l}\text { Test } \\
\text { No. }\end{array}$} & \multirow{2}{*}{$\begin{array}{c}\text { Irreversible } \\
\text { (IR) fouling } \\
\text { Rate } \\
(\mathrm{kPa} / \mathrm{day})\end{array}$} & \multirow[b]{2}{*}{$\begin{array}{c}\text { IR } \\
\text { fouling } \\
\mathrm{R}^{2} \text { value }\end{array}$} & \multirow[b]{2}{*}{$\begin{array}{l}\text { Filtration } \\
\text { time } \\
\text { (days) }\end{array}$} & \multirow[b]{2}{*}{$\begin{array}{c}\text { Water } \\
\text { Source }\end{array}$} & \multirow[b]{2}{*}{$\begin{array}{c}\text { Turbidity } \\
\text { (NTU) }\end{array}$} & \multicolumn{7}{|c|}{ LC-OCD data } \\
\hline & & & & & & $\begin{array}{c}\text { TOC } \\
(\mu \mathrm{g} / \mathrm{l})\end{array}$ & $\begin{array}{l}\text { DOC } \\
(\mu \mathrm{g} / \mathrm{l})\end{array}$ & $\begin{array}{c}\text { CDOC } \\
(\mu \mathrm{g} / \mathrm{l})\end{array}$ & $\begin{array}{c}\text { BP } \\
(\mu \mathrm{g} / \mathrm{l})\end{array}$ & $\underset{(\mu \mathrm{g} / \mathrm{l})}{\mathrm{HS}}$ & $\begin{array}{c}\text { BB } \\
(\mu \mathrm{g} / \mathrm{l})\end{array}$ & $\begin{array}{c}\text { LMW- } \\
\mathrm{N} \\
(\mu \mathrm{g} / \mathrm{l})\end{array}$ \\
\hline \multirow{3}{*}{1} & \multirow{3}{*}{3.6} & \multirow{3}{*}{0.86} & \multirow{3}{*}{5.88} & Raw & 0.58 & 1863 & 1751 & 1668 & 143 & 1003 & 269 & 254 \\
\hline & & & & SIX & 0.75 & 727 & 677 & 565 & 107 & 243 & 82 & 134 \\
\hline & & & & CMF & 0.10 & 552 & 540 & 454 & 45 & 214 & 75 & 120 \\
\hline \multirow{3}{*}{2} & \multirow{3}{*}{47.7} & \multirow{3}{*}{0.90} & \multirow{3}{*}{$1.74[\mathrm{~S}]$} & Raw & 2.10 & 2529 & 2372 & 2372 & 402 & 1276 & 356 & 338 \\
\hline & & & & SIX & 1.80 & 1080 & 1023 & 1020 & 287 & 409 & 114 & 210 \\
\hline & & & & CMF & $<0.1$ & 642 & 603 & 547 & 55 & 223 & 101 & 168 \\
\hline \multirow{3}{*}{3} & \multirow{3}{*}{6.2} & & & Raw & 2.00 & 3974 & 3801 & 3538 & 232 & 2429 & 458 & 419 \\
\hline & & 0.97 & 5.33 & Clarified & 0.99 & 994 & 937 & 924 & 51 & 351 & 291 & 231 \\
\hline & & & & CMF & $<0.1$ & 973 & 936 & 826 & 35 & 342 & 262 & 187 \\
\hline & & & & Raw & 1.70 & 1863 & 1786 & 1677 & 272 & 855 & 303 & 248 \\
\hline & & & & Clarified & 0.33 & 843 & 738 & 659 & 47 & 359 & 87 & 167 \\
\hline 4 & 0.7 & 0.87 & 5.47 & $\mathrm{C}+\mathrm{S}$ & 0.42 & 492 & 459 & 377 & 43 & 52 & 170 & 113 \\
\hline & & & & CMF & 0.29 & 473 & 441 & 358 & 37 & 43 & 146 & 132 \\
\hline & & & & Raw & 0.57 & 2288 & 2273 & 2297 & 124 & 1606 & 284 & 282 \\
\hline 5 & & & 501 & SIX & 0.60 & 1516 & 1519 & 1440 & 113 & 935 & 154 & 238 \\
\hline 5 & 6.1 & 0.99 & 5.91 & ILCA & 0.86 & 401 & 303 & 371 & 21 & 41 & 144 & 165 \\
\hline & & & & CMF & $<0.1$ & 364 & 311 & 346 & 32 & 46 & 121 & 147 \\
\hline & & & & Raw & 0.69 & 2060 & 1917 & 1976 & 188 & 1194 & 303 & 290 \\
\hline & & & 3.92 & SIX & 0.8 & 922 & 883 & 773 & 161 & 323 & 116 & 172 \\
\hline 6 & 5.4 & 0.96 & *WQ & ILCA & 1.4 & 563 & 425 & 356 & 30 & 47 & 161 & 119 \\
\hline & & & & $\mathrm{CMF}$ & 0.1 & 479 & 466 & 356 & 41 & 51 & 147 & 116 \\
\hline & & & & Raw & NS & 5188 & 5028 & 5248 & 365 & 3733 & 572 & 578 \\
\hline & & & & SIX & NS & 2742 & 2667 & 2810 & 321 & 1774 & 312 & 402 \\
\hline $6 \mathrm{a}$ & 571.4 & data & $0.27 * \mathrm{~S}$ & ILCA & NS & 2809 & 2560 & 2816 & 378 & 1679 & 297 & 460 \\
\hline & & & & CMF & NS & 951 & 906 & 835 & 71 & 408 & 148 & 208 \\
\hline & & & & Raw & 1.20 & 1524 & 1402 & 1484 & 274 & 740 & 239 & 232 \\
\hline & & & $3.94 * \mathrm{~W}$ & SIX & 1.40 & 747 & 642 & 661 & 220 & 223 & 91 & 127 \\
\hline 7 & 10.6 & 0.86 & Q & ILCA & 1.30 & 374 & 334 & 356 & 52 & 46 & 137 & 121 \\
\hline & & & & CMF & $<0.1$ & 393 & 331 & 316 & 44 & 45 & 116 & 111 \\
\hline & & & & Raw & 5.30 & 4150 & 4011 & 4279 & 443 & 2683 & 605 & 548 \\
\hline 8 & 38 & 095 & 533 & SIX & 5.50 & 1665 & 1555 & 1572 & 379 & 651 & 212 & 330 \\
\hline & & & 3.31 & ILCA & 5.90 & 1034 & 850 & 803 & 47 & 149 & 323 & 282 \\
\hline & & & & CMF & 0.13 & 966 & 855 & 777 & 40 & 148 & 313 & 276 \\
\hline & & & & Raw & 1.10 & 2890 & 2635 & 2623 & 181 & 1861 & 292 & 289 \\
\hline 9 & 2.1 & 0.83 & 5.6 & ILCA & 1.00 & 771 & 741 & 600 & 40 & 220 & 176 & 164 \\
\hline & & & & CMF & 0.31 & 707 & 645 & 520 & 27 & 161 & 182 & 150 \\
\hline & & & & Raw & 0.96 & 2787 & 2630 & 2592 & 184 & 1828 & 308 & 272 \\
\hline & & & & SIX & 1.00 & 2283 & 2117 & 2022 & 155 & 1370 & 274 & 223 \\
\hline 10 & 1.7 & 0.94 & 5.6 & ILCA & 1.60 & 599 & 471 & 433 & 35 & 139 & 126 & 133 \\
\hline & & & & CMF & $<0.1$ & 503 & 485 & 392 & 31 & 114 & 124 & 123 \\
\hline & & & & Raw & 0.74 & 2334 & 2245 & 2136 & 132 & 1452 & 297 & 254 \\
\hline 11 & 17 & 093 & 56 & SIX & 0.78 & 1812 & 1788 & 1660 & 121 & 1083 & 228 & 228 \\
\hline 11 & 1.7 & 0.93 & 3.6 & ILCA & 1.40 & 523 & 512 & 441 & 30 & 156 & 110 & 143 \\
\hline & & & & CMF & 0.17 & 471 & 435 & 359 & 25 & 114 & 96 & 123 \\
\hline & & & & Raw & 0.65 & 2187 & 2129 & 2040 & 119 & 1404 & 258 & 259 \\
\hline 12 & 1.4 & 0.93 & 5.6 & ILCA & 0.72 & 717 & 688 & 609 & 29 & 229 & 163 & 187 \\
\hline & & & & CMF & 0.11 & 550 & 512 & 458 & 26 & 139 & 147 & 147 \\
\hline & & & & Raw & 0.87 & & 2360 & & & & & \\
\hline 13 & Very high & fouling - & X TMP & SIX & 1.05 & & 1100 & & & & & \\
\hline & & & & CMF & 0.14 & & 850 & & & & & \\
\hline
\end{tabular}

[S] run stopped due to shut-down; WQ water quality change, NS not sampled, NTU nephelometric turbidity units, TOC total organic carbon, DOC dissolved organic carbon, CDOC chromatographically detectable DOC, BP biopolymers, HS humic substances, BB building blocks, LMW-N low molecular weight neutral. Italicised DOC values (Test 13) were inferred from UVT data. 


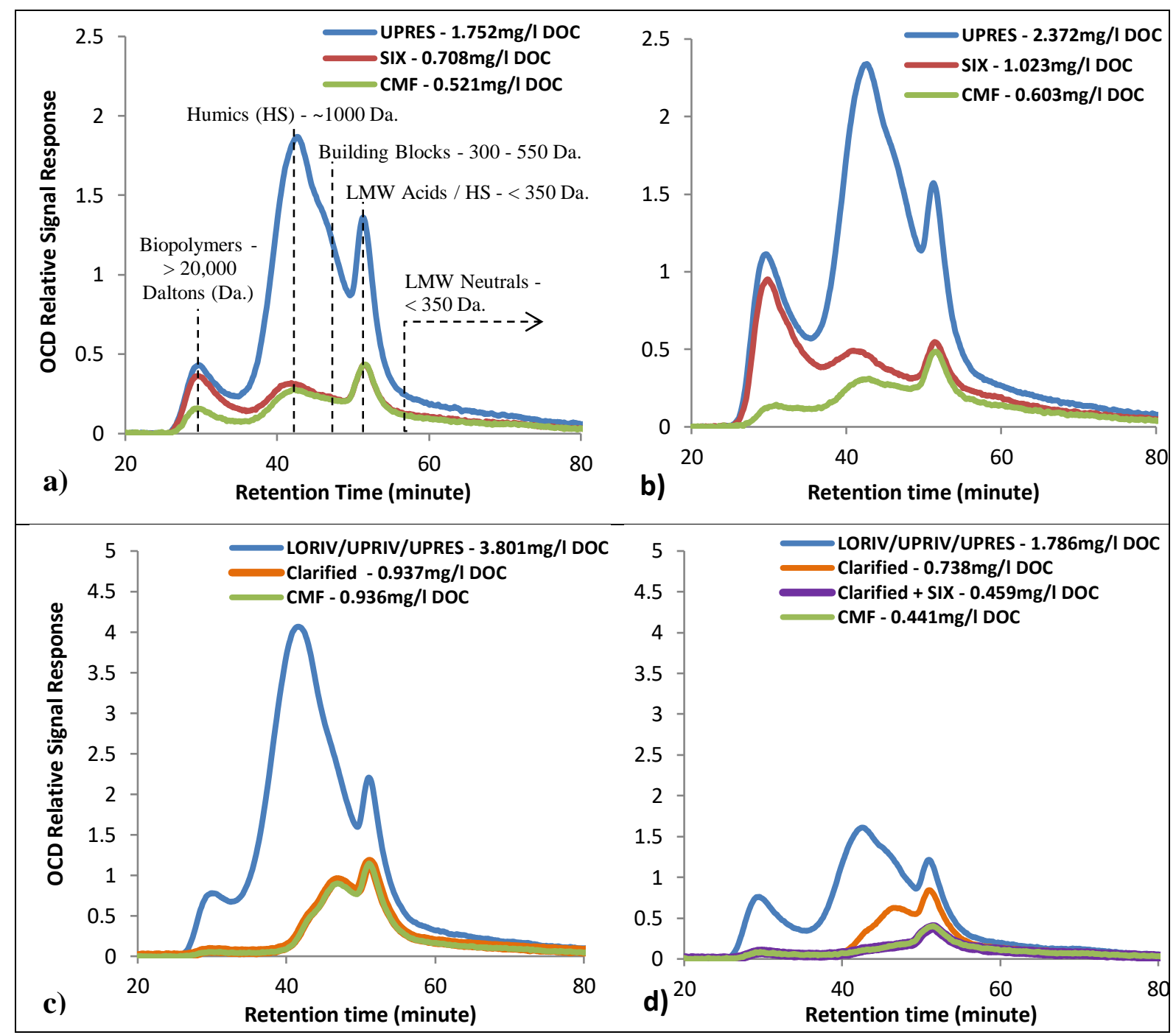

Figure 3: LC-OCD chromatograms a) Test 1: SIX only with low DOC UPRES raw water (fraction peak assignation shown for reference) b) Test 2 - SIX only with moderate DOC UPRES raw water c) Test 3 - Clarification only with moderate DOC blend of raw waters d) Test 4 - Clarification followed by SIX with low DOC blend of raw waters

For the lowest raw water organic concentrations (e.g. Table 2 - Test 1 ), fouling was effectively abated by the routine physical and chemical cleaning. The increased fouling recorded in Test 2 was associated with greater retention of HMW organics than for Test 1 (80\% vs. 58\% for $\mathrm{BP}$ ), corroborating previous work on the deleterious impact of increasing BP mass loads on irreversible fouling (Fabris et al. 2007; Fan et al. 2008; Zheng et al. 2010; Huang et al. 2012; Myat et al. 2013; Tian et al. 2013; Shang et al. 2015; Kimura et al. 2014). Whilst ion exchange can significantly reduce overall DOC concentrations, previous research indicates that it does not lead to significant reductions in membrane fouling due to the limited removal of the HMW organics (Humbert et al. 2007; Fabris et al. 2007; Fan et al. 2008; Huang et al. 2012). Analysis of the adsorbed organics revealed that a good correlation was obtained between membrane fouling and adsorption of (a) overall DOC $\left(\mathrm{R}^{2}=0.89\right)$, (b) BPs $\left(\mathrm{R}^{2}=0.95\right)$ and (c) HSs $\left(\mathrm{R}^{2}=\right.$ $0.98)$, whereas the correlations with LMW fractions were significantly weaker $\left(\mathrm{R}^{2}=0.14\right.$ $0.33)$.

Test 3, using fully clarified water from the full-scale plant (Fig. 3c), was performed under the same membrane operating conditions as Tests 1 and 2 (using SIX pretreatment) but whilst 
challenged with more highly fouling river water. Results indicated the removal of the HMW organics by clarification to provide a low-to-moderate fouling rate of $6.2 \mathrm{kPa} /$ day (Table 2 , Test 3). Whilst pre-clarification removed the LMW organics to a lesser extent than possible with SIX pretreatment (Fig. 3c), these organics were not retained by the membrane whereas the HMW organics were largely retained following SIX pretreatment. Following coagulation pretreatment no adsorption was detected. In Test 4 the clarified water was further treated by SIX (under the same operational conditions as Tests 1-3) to establish if removing additional LMW organics from the clarified water, would further suppress membrane fouling. The lower fouling rate of $0.9 \mathrm{kPa} /$ day (Table 2, Test 4) was associated with negligible organics adsorption $(0.018 \mathrm{mg} / \mathrm{l}$ DOC. Fig. 3d), although the apparent adsorption recorded was higher than in Test 3.

Further tests comparing clarification only with clarification + SIX using the same operational conditions as Tests 1-4 at a higher applied flux of $175 \mathrm{LMH}$, indicated a moderate fouling rate of $11 \mathrm{kPa} /$ day for clarification alone and a much lower rate of $2.4 \mathrm{kPa} /$ day for clarification + SIX, despite a significantly higher raw water DOC. As with Test 3 and 4, DOC adsorption was negligible (-0.022 vs. $0.037 \mathrm{mg} / \mathrm{L}$ for clarification vs. clarification + SIX). This suggests when fouling was low, the fouling was related to factors other than organic adsorption, such as floc characteristics (Jarvis et al., 2008). It is also possible that the ion exchange material adsorbs any residual anionic polyelectrolyte from the clarification process, which would otherwise affect membrane fouling (Wang et al. 2011; Yu et al. 2013). Two further tests with clarification + SIX pre-treatment performed with the same operational conditions as Tests 1-4, but at higher fluxes of 200 and $250 \mathrm{LMH}$, yielded fouling rates of 5.5 and $197 \mathrm{kPa} /$ day respectively, suggesting that the highest value exceeded the critical flux.

\subsection{SIX/LCA pre-treatment with enhanced CEB, manual control (Tests 5-8)}

Jar tests confirmed contact times below two minutes to be sufficient for flocculating residual HMW organics following SIX treatment of the three raw waters, corroborating work performed by Meyn et al. (2012) on ILCA upstream of membrane filtration. Subsequent pilot trials were conducted with SIX followed by $\mathrm{pH}$ correction and 2-4 minutes ILCA at relatively low coagulant doses (50-90\% less than that employed for the full-scale clarification process), permitted by the removal of a large amount of DOC by SIX (Huang et al. 2012; Humbert et al. 2007; Jarvis et al. 2008). A flux of $185 \mathrm{LMH}$ was employed for these trials, with a $\mathrm{NaOH}-$ based CEB: supplementary trials revealed $\mathrm{NaOH}$ to be more effective than $\mathrm{NaOCl}$ for CEB.

Analysis of the organic content of the three raw water types used for these tests revealed it to be dominated by aromatic organics (high SUVA) with all organic fractions, including BPs, adsorbing UV strongly. The UVT could thus be used as a relatively accurate surrogate measure of DOC, confirmed by the strong correlation $\left(\mathrm{R}^{2}=0.92\right)$ between UVT and the LC-OCDdetermined DOC. The algorithm from jar test data (Equation 1) was used to manually set the coagulant dose based on the feed water UVT. This generally led to negligible adsorption of organic matter on the membrane and corresponding low fouling rates. High observed membrane fouling, following rapid changes in raw water quality and subsequent sub-optimal coagulation conditions, was accompanied by measurable organics adsorption.

Results from Tests 5-8 (Table 2) indicated that for optimised, manually-controlled coagulant dosing and $\mathrm{pH}$ adjustment, membrane fouling was low to moderate $(3.8-11 \mathrm{kPa} /$ day $)$ at the high flux of $185 \mathrm{LMH}$. The DOC removal by the SIX ILCA CMF process was similar to that attained in the scoping trials based on full-scale clarification followed by SIX, i.e. 74-88\% 
relative to the raw water. As with the scoping trials with clarification alone and clarification + SIX (Table 1-2, Tests 3 and 4), changes in fouling rate could not be related to the LC-OCD data as membrane organic adsorption was negligible when coagulation conditions were optimised. Further tests with the same operation conditions as Tests 5-8, but at a lower flux of $112 \mathrm{LMH}$, yielded low fouling rates of 1.0-1.7/day. Instances of non-optimum coagulation conditions, due to rapid changes in raw water quality, always led to rapid fouling which was reflected by measurable organics adsorption onto the membrane (Table 2, Test 6a). These results demonstrate the critical nature of optimal pre-coagulation in direct membrane filtration.

\subsection{IEX dose trials in combination with ILCA (Tests 9-13)}

Tests conducted at $109 \mathrm{LMH}$ on standard, reduced or zero SIX pretreatment yielded low fouling rates of 1.4-2.1 kPa/day at doses of 0 to $18 \mathrm{~mL} / \mathrm{L}$ SIX (Table 1-2, Tests 9-12), with optimised coagulant dosing. Coagulant demand increased with decreasing SIX dose, but under these optimised coagulation conditions there was no correlation between fouling and SIX dose with the fouling rate remaining stable at 0,9 or $18 \mathrm{~g} / \mathrm{l}$ resin doses. However, when the ILCA was taken off line and SIX pretreatment alone was employed, rapid fouling was observed (Table 1-2, Test 13). This confirmed the relative importance to membrane fouling of enmeshment of HMW BP organics within coagulant flocs, compared to the additional removal of LMW fractions by SIX. In contrast to the full clarification or clarification and SIX tests (Section 3.1), the addition of SIX to ILCA pretreatment yielded no appreciable fouling suppression. This was possibly due to either the change in $\mathrm{CEB}$ reagent (from $\mathrm{NaOCl}$ to $\mathrm{NaOH}$ ), the difference in floc morphology, or, most likely, the nature of the direct filtration process where a cake is formed on the membrane surface. Previous research has suggested that this cake layer may reduce irreversible membrane fouling by either adsorbing some unflocculated organics, and therefore preventing their adsorption to the membrane (Dong et al. 2007), or by rejecting fine flocs or colloids which would otherwise plug the membrane pores (Guigui et al. 2002).

\subsection{SIX + ILCA pretreatment with automated coagulant and $\mathrm{pH}$ control}

A virgin membrane and an automated $\mathrm{pH}$ and coagulant control system was installed prior to an extended fouling rate test (21 days) at a high flux of $185 \mathrm{LMH}$ on the UPRIV source water (Fig. 4). Low fouling rates were sustained despite rapid changes in raw water quality and DOC concentration associated with heavy rainfall events. Cessation of coagulation during this trial (Day 9) led to a rapid increase in the TMP, which was ameliorated on reinstating the coagulant dose, albeit at a slightly higher baseline TMP. Taking the SIX dosing off line on Day 13 did not lead to an increased fouling rate, a stable TMP being maintained. Overall a very low fouling rate of $0.3 \mathrm{kPa}$ /day was sustained despite coagulant dose upset, regardless of the resin dose, whilst operating at high flux on variable quality raw water. The results further corroborate coagulation as being the most important pre-treatment for suppressing fouling and confirmed that very low fouling rates were possible at high fluxes when operating with automated coagulant dosing and $\mathrm{pH}$ control. The high permeability of the virgin membrane was sustained throughout the trial. Further tests of around 2 months' duration yielded an overall fouling rate $0.24 \mathrm{kPa} /$ day, with a subsequent CIP returning the membrane permeability back to that of the virgin material. 


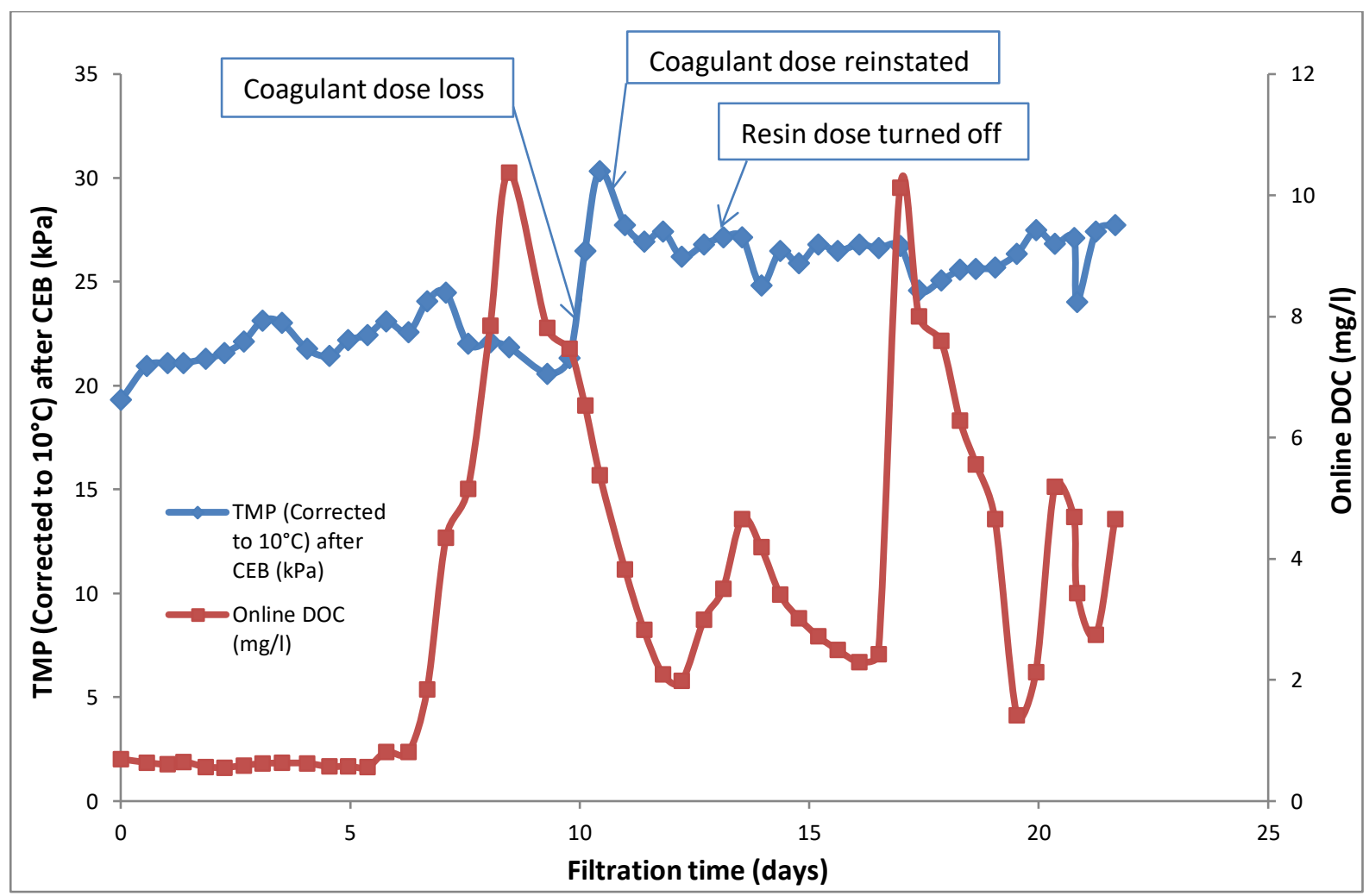

Figure 4: $\quad$ Extended operation during spate conditions (UPRIV) with automated coagulant and $\mathrm{pH}$ control

\section{Conclusions}

A pilot-scale study of the efficacy of pretreatment for ceramic membrane filtration of surface waters as applied to potable water supply has revealed:

- Suspended ion exchange (SIX) resin removed predominantly low molecular weight (LMW) organic matter whereas coagulation removed the high molecular weight (HMW) fractions. The combination of full clarification (coagulation with floc blanket) and SIX led to substantial removal of all organic fractions to leave a low residual dissolved organic carbon (DOC) concentration.

- The HMW organics (present following SIX), including biopolymers, were retained by the membrane and caused rapid fouling, whereas LMW organics (present following coagulation alone) were not retained when coagulation was optimised.

- When using optimised in-line coagulation (ILCA) with direct membrane filtration, as opposed to full clarification pretreatment, additional pretreatment with SIX provided no measureable benefit with regards to membrane fouling suppression.

- Sub-optimal coagulation conditions, such as under-dosing of coagulant or inappropriate $\mathrm{pH}$ adjustment, resulted in rapid fouling.

- SIX ILCA pretreatment provided similar DOC removal to clarification followed by SIX pretreatment whilst providing a more compact, efficient and flexible process than clarification and SIX pretreatment.

- A fully optimised system with automated $\mathrm{pH}$ and coagulant control allowed operation at an elevated flux of $185 \mathrm{LMH}$ on a water source of highly variable organic concentration with overall membrane fouling rates below $0.3 \mathrm{kPa}$ /day over a two-month test period. 


\section{Acknowledgements}

The authors would like to acknowledge: South West Water, Exeter, U.K who funded and supported the work and South West Water Laboratories, Exeter, U.K for the provision of water analysis.

Gilbert Galjaard, Holly Shorney-Darby, Jumeng Zheng and Harry Scheerman from PWN Technologies, Netherlands for the design and build of the pilot plant and for ongoing technical support throughout the project.

RWB Water Services, Almelo, Netherlands for the design, build and ongoing assistance with maintenance of the pilot plant.

Het Water Laboratorium, Haarlem, Netherlands who provided LC-OCD analysis.

\section{References}

Bond, T., Goslan, E.,H., Parsons, S.,A., Jefferson, B., 2011. Treatment of disinfection byproduct precursors. Environmental Technology, 32 (1), pp. 1-25.

Dong, B., Dong, B., Gao, N., Deng, H., Fan, J., 2005. Effect of coagulation on preventing membrane from fouling. Huanjing Kexue/Environmental Science, 26(1), pp. 90-93.

Fabris, R., Lee, E.K., Chow, C.W.K., Chen, V., Drikas, M., 2007. Pre-treatments to reduce fouling of low pressure micro-filtration (MF) membranes. Journal of Membrane Science, 289(1-2), pp. 231-240.

Fan L., Nguyen T., Roddick, F.A., Harris, J.L., 2008. Low-pressure membrane filtration of secondary effluent in water reuse: Pre-treatment for fouling reduction. Journal of Membrane Science, 320(1-2), pp. 135-142.

Fearing, D.A., Banks, J., Guyetand, S., Eroles, C.M., Jefferson, B., Wilson, D., Hillis, P., Campbell, A.T., Parsons, S.A., 2004. Combination of ferric and MIEX® for the treatment of a humic rich water. Water Research, 38 (10), pp. 2551-2558.

Freeman, S., Shorney-Darby, H., 2011. What's the buzz about ceramic membranes? Journal of the American Water Works Association. 103, pp. 12-13.

Galjaard, G., Martijn, B., Koreman, E., Bogosh, M., Malley, J., 2011. Performance Evaluation SIX ${ }^{\circledR}$-CeraMac ${ }^{\circledR}$ in Comparison with Conventional Pre-treatment Techniques for Surface Water Treatment. Water Practice and Technology, 6 (4).

Gray, S.R., Dow, N., OrbellL, J.D., Tran, T., Bolto, B.A., 2011. The significance of interactions between organic compounds on low pressure membrane fouling. Water Science and Technology, 64(3), pp. 632-639.

Guigui, C., Rouch, J.C., Durand-Bourlier, L., Bonnelye, V., Aptel, P., 2002. Impact of coagulation conditions on the in-line coagulation / UF process for drinking water production. Desalination, 147(1-3), pp. 95-100.

Hofs, B., Ogier, J., Vries, D., Beerendonk, E.F., Cornelissen, E.R., 2011. Comparison of ceramic and polymeric membrane permeability and fouling using surface water, Separation and Purification Technology, 79, p 365- 374

Huang, H., Schwab, K., Jacangelo, J.G., 2009. Pretreatment for low pressure membranes in water treatment: A review. Environmental Science and Technology, 43(9), pp. 3011-3019.

Huang, H., Cho, H., Schwab, K.J., Jacangelo, J.G., 2012. Effects of magnetic ion exchange pretreatment on low pressure membrane filtration of natural surface water. Water research, 46(17), pp. 5483-5490. 
Huber, S.A., Balz, A., Abert, M., Pronk, W., 2011. Characterisation of aquatic humic and nonhumic matter with size-exclusion chromatography - organic carbon detection - organic nitrogen detection (LC-OCD-OND). Water Research, 45 (2), pp. 879-885.

Humbert, H., Gallard, H., Jacquemet, V., Croué , J., 2007. Combination of coagulation and ion exchange for the reduction of UF fouling properties of a high DOC content surface water. Water Research. 41 (17), pp. 3803-3811.

Jarvis, P., Mergen, M., Banks, J., Mcintosh, B., Parsons, S.A., Jefferson, B., 2008. Pilot scale comparison of enhanced coagulation with magnetic resin plus coagulation systems. Environmental Science and Technology, 42 (4), pp. 1276-1282.

Kimura, K., Tanaka, K., Watanabe, Y., 2014. Microfiltration of different surface waters with/without coagulation: Clear correlations between membrane fouling and hydrophilic biopolymers. Water Research, 49, pp. 434-443.

Lai, C., Chou, Y., Yeh, H., 2015. Assessing the interaction effects of coagulation pretreatment and membrane material on UF fouling control using HPSEC combined with peak-fitting. Journal of Membrane Science, 474, pp. 207-214.

Lee, S. and Kim, J., 2014. Differential natural organic matter fouling of ceramic versus polymeric ultrafiltration membranes. Water research, 48(1), pp. 43-51.

Mergen, M.R.D., Adams, B.J., Vero, G.M., Price, T.A., Parsons, S.A., Jefferson, B., Jarvis, P., 2009. Characterisation of natural organic matter (NOM) removed by magnetic ion exchange resin (MIEX® Resin) Water Science and Technology: Water Supply, 9 (2), pp. 199-205.

Metcalfe D., Rockey C., Jefferson B., Judd S., Jarvis P., 2015. Removal of disinfection byproduct precursors by coagulation and an innovative suspended ion exchange process. Water research, 87, pp. 20-28.

Meyn, T., Altmann, J., Leiknes, T., 2012. In-line coagulation prior to ceramic microfiltration for surface water treatment-minimisation of flocculation pre-treatment. Desalination and Water Treatment, 42 (1-3), pp. 163-176.

Myat, D.T., Mergen, M., Zhao, O., Stewart, M.B., Orbell, J.D., Gray, S., 2012. Characterisation of organic matter in IX and PACl treated wastewater in relation to the fouling of a hydrophobic polypropylene membrane. Water research, 46(16), pp. 5151-5164.

Singer, P.C., Bilyk, K., 2002. Enhanced coagulation using a magnetic ion exchange resin. Water Research, 36 (16), pp. 4009-4022.

Subhi, N., Leslie, G., Chen, V., Le-clech, P., 2012. Organic Fouling of Ultrafiltration Membrane: Detailed Characterization by Liquid Chromatography with Organic Carbon Detector (LC-OCD). Separation Science and Technology, 48(2), pp. 199-207.

Shang, R., Vuong, F., Hu, J., Li, S., Kemperman, A.J.B., Nijmeijer, K., Cornelissen, E.R., Heijman, S.G.J., Rietveld, L.C., 2015. Hydraulically irreversible fouling on ceramic MF / UF membranes: Comparison of fouling indices, foulant composition and irreversible pore narrowing. Separation and Purification Technology, 147, pp. 303-310.

Tian, J., Ernst, M., Cui, F., Jekel, M., 2013. Correlations of relevant membrane foulants with UF membrane fouling in different waters. Water research, 47(3), pp. 1218-1228.

Vreeburg J.H.G., Schippers D., Verberk J.Q.J.C., Van Dijk J.C., 2008. Impact of particles on sediment accumulation in a drinking water distribution system. Water research, 42(16), pp. 4233-4242. 
Wang, S., Liu, C., Li, Q., 2011. Fouling of microfiltration membranes by organic polymer coagulants and flocculants: Controlling factors and mechanisms. Water research, 45(1), pp. 357-365.

Yu, W., Liu, H., Xu, L., Qu, J., Graham, N., 2013. The pre-treatment of submerged ultrafiltration membrane by coagulation - Effect of polyacrylamide as a coagulant aid. Journal of Membrane Science, 446, pp. 50-58.

Zheng, X., Ernst, M., Huck, P.M., Jekel, M., 2010. Biopolymer fouling in dead-end ultrafiltration of treated domestic wastewater. Water research, 44(18), pp. 5212-5221. 